\title{
ELISA-IgM para diagnóstico da esquistossomose mansoni em área de baixa endemicidade
}

\author{
IgM-ELISA for diagnosis of schistosomiasis \\ mansoni in low endemic areas
}

Edward José de Oliveira 1

Hermínia Yoshio Kanamura 1

Luiz Cândido de Souza Dias 2

Lanny Cristina Burlandy Soares 2

Dirce Mary Correia Lima 3

Ricardo Mário de Carvalho Ciaravolho 4

\footnotetext{
1 Departamento de Análises Clínicas e Toxicológicas, Faculdade de Ciências Farmacêuticas, Universidade de São Paulo. Av. Lineu Prestes 580, São Paulo, SP 05508-900, Brasil. edwardoliveira@bol.com.br edward@fcm.unicamp.br kanamura@usp.br

2 Departamento de Patologia Clínica, Faculdade de Ciências Médicas, Universidade Estadual de Campinas. C. P. 6111, Campinas, $S P$ 13083-970, Brasil.

luizcan@fcm.unicamp.br

3 Instituto de Medicina Tropical de São Paulo, Faculdade de Ciências Médicas, Universidade de São Paulo. Av. Dr. Enéas de Carvalho Aguiar 470, São Paulo, SP 05403-000, Brasil.

4 Superintendência de Controle de Endemias, Secretaria de Saúde do Estado de São Paulo. Rua Paula Souza 166, São Paulo, SP 01027-000, Brasil.
}

\begin{abstract}
An immunoenzymatic method for the detection of IgM antibodies (IgM-ELISA) against a fraction of Schistosoma mansoni adult worm antigen, soluble in trichloroacetic acid (TCA-soluble fraction), was evaluated for epidemiological purposes in low endemic areas for schistosomiasis. Blood samples on filter paper were collected from a population living in the municipality of Pedro de Toledo, São Paulo State, and submitted to IgM-ELISA. The results were compared to those obtained by the IgM-immunofluorescence test (IgM-IFT) and the Kato-Katz parasitological method. Positive rates of $36.8 \%, 33.5 \%$, and $1.6 \%$ were obtained respectively by the IgM-ELISA, IgM-IFT, and Kato-Katz methods. The geometric mean obtained by the parasitological method was 40.9 eggs per gram of feces (epg). The nearly perfect agreement observed between the two serological tests (Kappa index of 0,89) indicates good diagnostic performance by the evaluated test. IgM-ELISA is a potentially useful method for diagnosis of schistosomiasis in individuals with low worm burden.
\end{abstract}

Key words Schistosoma Mansoni; Enzyme-Linked Immunosorbent Assay; Antibodies; Schistosomiasis; Serology

Resumo Um método imunoenzimático para detecção de anticorpos IgM (ELISA-IgM) contra uma fração do extrato total de vermes de Schistosoma mansoni, solúvel em ácido tricloro acético (fração TCA-solúvel) foi avaliado para fins epidemiológicos, em área de baixa endemicidade para esquistossomose. Amostras de sangue em papel de filtro, coletadas de uma população residente no município de Pedro de Toledo, Estado de São Paulo, foram submetidas ao ELISA-IgMe os resultados, analisados comparativamente aos obtidos pela RIFI-IgM e pelo exame parasitológico de fezes Kato-Katz. Obteve-se 36,8\% de positividade pelo ELISA-IgM, 33,5\% pela RIFI-IgM e 1,6\% pelo Kato-Katz, que indicou uma média geométrica de 40,9 ovos por grama de fezes (opg). A concordância de resultados, quase perfeita (índice Kappa de 0,89), observada entre os dois métodos sorológicos, indica um bom desempenho diagnóstico do teste em avaliação. O ELISA-IgM constitui-se em um método potencialmente útil para fins diagnósticos da esquistossomose, em indivíduos com baixa carga parasitária.

Palavras-chave Schistosoma Mansoni; Ensaio Imunoadsorvente Enzima-Associado; Anticorpos; Esquistossomose; Sorologia 


\section{Introdução}

A partir da implantação da Campanha de Combate à Esquistossomose (CACESQ) pela Secretaria da Saúde do Estado de São Paulo, em 1968, o método parasitológico de fezes, segundo a técnica de Kato-Katz (Katz et al., 1972), vem sendo utilizado como método único para selecionar os indivíduos a serem submetidos à quimioterapia. No entanto, nas áreas onde a doença é de pouca gravidade, com manifestações leves e pouco específicas, com a maioria dos portadores eliminando menos de 100 ovos do parasita por grama de fezes, como acontece nas áreas endêmicas do Estado de São Paulo, a prevalência real da doença fica subestimada, quando se emprega somente esse método para diagnosticar a esquistossomose (De Vlass \& Gryssels, 1992; Dias et al., 1992; Noya et al., 1999). Isso ocorre por causa da baixa sensibilidade do método de KatoKatz, que depende diretamente da quantidade de fezes examinadas e do número de ovos eliminados pelo portador (Barreto et al., 1990; De Vlass \& Gryssels, 1992; Engels et al., 1996).

Estudos epidemiológicos realizados em áreas consideradas de baixa endemicidade para esquistossomose no Estado de São Paulo, possibilitaram verificar a manutenção de prevalências residuais, causadas possivelmente por diversos fatores inerentes ao controle da doença (Dias et al., 1989, Marçal Jr. et al., 1993). Entre outros, um dos fatores que pode estar contribuindo para a manutenção da parasitose é a relativa falta de sensibilidade do método parasitológico de fezes na detecção de indivíduos com baixa carga parasitária. Sendo assim, o emprego de metodologias diagnósticas alternativas, que permitam estabelecer índices de prevalência mais próximos da verdadeira, poderá contribuir muito para o sucesso do controle da doença nessas áreas.

A reação de imunofluorescência indireta em cortes parafinados de vermes adultos para pesquisa de anticorpos IgM (RIFI-IgM) contra antígenos do tubo digestivo (Silva et al., 1992) vem sendo utilizada como método complementar para diagnóstico individual da esquistossomose nos órgãos de saúde pública do Estado de São Paulo. Essa técnica também tem sido avaliada em grupos de crianças em idade escolar residentes em áreas de baixa endemicidade nesse Estado e tem demonstrado índices de sensibilidade e especificidade que variam de $95 \%$ a $98 \%$ quando comparados ao método parasitológico de Kato-Katz (Kanamura et al., 1998a, 1998b; Lima et al., 1998). No entanto, a leitura subjetiva, a necessidade de um microscópio de fluorescência e a impossibilidade de automação di- ficultam o emprego dessa técnica em levantamentos epidemiológicos mais amplos.

O método de ELISA, por possibilitar a automação, além de proporcionar ensaios quantitativos, mostra-se mais bem adaptado para aplicação em estudos populacionais. Entretanto, sua utilização no nosso meio é ainda muito limitada, devido sobretudo à falta de reagentes comerciais aplicáveis ao diagnóstico da esquistossomose. Variantes técnicas têm sido empregadas para detecção de anticorpos contra antígenos de vermes adultos (Sathe et al., 1991; Silva et al., 1998; Valli et al., 1997) ou de ovos (Doenhoff et al., 1993; Eltiro et al., 1992; Kamal et al., 1994; Tanabe et al., 1990), de Schistosoma mansoni (Sambon, 1907). No entanto, alguns pesquisadores verificaram a presença de reações cruzadas em amostras de soro de indivíduos infestados com outros parasitas que não o $S$. mansoni, quando se utiliza extrato total como antígeno no método de ELISA e Western-blot para pesquisa de anticorpos IgG (Correia-Oliveira et al., 1988; Valli et al., 1997). Em recente trabalho, em que o método de ELISA foi utilizado para detecção de anticorpos IgG contra o extrato total de vermes adultos, os resultados obtidos demonstraram falta de concordância quando comparados à reação de imunofluorescência indireta (RIFI) para pesquisa de anticorpos IgM em cortes parafinados de vermes adultos (Silva et al., 1998).

A utilização no ELISA de uma fração do extrato total de vermes adultos, solúvel em ácido tricloro acético (fração TCA-solúvel), purificada por Deelder et al. (1976), permitiu padronizar um teste imunoenzimático para pesquisa de anticorpos IgM (ELISA-IgM) que apresentou resultados comparáveis à RIFI-IgM. Embora o método de ELISA-IgM tenha fornecido bons resultados quando aplicado para diagnóstico da esquistossomose no exterior (Deelder \& Kornelis, 1980), estudos de avaliação fazem-se necessários para adaptá-lo às nossas condições laboratoriais e ambientais. No presente trabalho, o método de ELISA-IgM foi padronizado e empregado com a finalidade de verificar seu desempenho como ferramenta diagnóstica em levantamentos epidemiológicos em áreas de baixa endemicidade para esquistossomose, como ocorre em algumas áreas do município de Pedro de Toledo, no Estado de São Paulo.

\section{Material e métodos}

\section{Característica da população}

Para este estudo foi escolhida a população do Município de Pedro de Toledo. Dessa popula- 
ção foram selecionados dois grupos: um grupo de indivíduos de todas as idades, de ambos os sexos, residentes em áreas urbana e rural do município, expostos ao risco de infestação por S. mansoni, denominado Grupo de Estudo (GE); e outro constituído de 50 indivíduos, de todas as idades e de ambos os sexos, residentes em área não endêmica para esquistossomose (Vila São Lourenço), portadores de outras infestações parasitárias que não a esquistossomose, denominado Grupo Controle (GC). No GE, do total de 1.117 indivíduos selecionados por meio da técnica de amostragem aleatória simples, utilizando o programa Epi Info, 897 forneceram alguma amostra biológica. Foram coletadas amostras de fezes de 749 participantes e amostras de sangue de 591, tendo-se obtido as duas amostras biológicas somente de 481 indivíduos. No GC foram obtidas amostras de sangue de todos os 50 participantes e amostras de fezes de 37 .

\section{Amostras de sangue}

Durante o segundo semestre de 1998, as amostras de sangue foram coletadas em papel de filtro Whatman, no 3, cortado em tiras de $1 \mathrm{~cm}$ de largura por $10 \mathrm{~cm}$ de comprimento. Pedaços com área de $1 \mathrm{~cm}^{2}$ foram mergulhados e macerados em $330 \mu \mathrm{L}$ de solução salina tamponada com fosfatos (SSTF) e mantidos em repouso por 18 a 24 horas no refrigerador, de forma a obterse um eluato (sangue eluído) que corresponderia aproximadamente à diluição de 1/20 do soro (Ferreira \& Carvalho, 1982; Silva et al., 1998).

\section{Amostras de fezes}

Cada indivíduo coletou uma amostra de fezes em recipientes (latinhas) deixados previamente em suas residências no mesmo período indicado acima. Foram preparadas três lâminas por amostra fecal, segundo a técnica de Kato-Katz (Katz et al., 1972). Uma vez que a equipe de enfermagem do posto de saúde da Vila São Lourenço não estava treinada para montar lâminas pela técnica de Kato-Katz, as amostras de fezes do GC foram coletadas em recipientes Coprotest ${ }^{\circledR}$. As amostras foram enviadas para nosso laboratório e processadas seguindo o protocolo do Coprotest ${ }^{\circledR}$ (Cerqueira, 1988).

\section{ELISA-IgM}

Foi realizado segundo técnica descrita por Deelder \& Kornelis (1980), com algumas modificações. Para execução do método, placas de poliestireno de fundo plano (Nunc, Brand Pro- ducts, Dinamarca) foram sensibilizadas com a fração TCA-solúvel do extrato total de $S$. mansoni. O eluato a $1 / 20$, obtido como descrito anteriormente, foi diluído cinco vezes em SSTF, contendo Tween-20 a $0,05 \%$ e leite desnatado a $1 \%$, de modo a obter-se uma diluição correspondente a $1 / 100$. Utilizou-se conjugado anti-IgM-humano-peroxidase (Sigma Chemical Company, St. Louis, Estados Unidos) diluído a 1/4.000 e, como mistura cromógena, peróxido de hidrogênio e ortofenilenodiamina, sendo a reação interrompida com solução $1 \mathrm{~N}$ de $\mathrm{H}_{2} \mathrm{SO}_{4}$. As amostras foram ensaiadas em triplicata. Como controle utilizou-se, em duplicata, diluição seriada de soro-padrão positivo de título conhecido e oito eluatos negativos obtidos a partir de amostras de sangue de indivíduos comprovadamente não esquistossomóticos. A leitura da reação foi realizada em leitora de microplaca (SLT-Labinstruments-Spectra I), em comprimento de onda de $492 \mathrm{~nm}$. Foram consideradas positivas as amostras cujas médias aritméticas das leituras de absorbância foram superiores ao limiar de reatividade (cutoff). Esse foi determinado, em cada dia de reação, pela média aritmética das leituras de absorbância dos oito eluatos negativos, acrescida de dois desvios-padrão.

\section{Reação de Imunofluorescência} Indireta (RIFI-IgM)

Foi realizada segundo técnica descrita por Silva et al. (1992). O eluato foi utilizado diluído a 1/20 e o conjugado fluorescente anti-IgM (Biolab, Rio de Janeiro, Brasil), diluído a 1/320, em SSTF contendo 0,25mg\% de azul de Evans. As amostras foram ensaiadas em duplicata e, como controle, foi avaliada, em cada dia de reação, uma diluição seriada de soro-padrão positivo, de título conhecido, e um eluato negativo obtido a partir de sangue de indivíduo comprovadamente não esquistossomótico. As lâminas foram examinadas em aumento de 100 vezes, utilizando-se microscópio de fluorescência (Olympus BX-FLA) dotado de epifluorescência com lâmpada de mercúrio de $100 \mathrm{~W}$, filtro de excitação BP 450-480 e filtro de barreira BA 515. Foram consideradas como positivas as amostras que apresentaram fluorescência nas estruturas relacionadas ao tubo digestivo do verme.

\section{Exame coproparasitológico}

As amostras fecais do GE foram processadas pela técnica de Kato-Katz (Katz et al., 1972), e as do GC foram preparadas segundo o protocolo do kit comercial Coprotest ${ }^{\circledR}$ (Cerqueira, 1988), tendo sido preparadas duas lâminas de cada 
amostra, examinadas em microscópio óptico comum em aumento de 100 vezes.

\section{Análise estatística}

Os resultados obtidos pelos métodos imunológicos e pelo exame parasitológico de fezes foram inseridos no programa Epi Info, versão 6.04, da Organização Mundial da Saúde (OMS). Por meio desse programa foram calculados os coeficientes Kappa e os intervalos de confiança.

\section{Aspectos éticos}

O presente trabalho obteve parecer favorável do Comitê de Ética em Pesquisa do Instituto de Medicina Tropical de São Paulo e do Comitê de Ética em Pesquisa da Faculdade de Ciências Farmacêuticas da Universidade de São Paulo.

\section{Resultados}

A positividade parasitológica no GE foi de 1,6\% (12/749) para S. mansoni, 23,4\% (175/749) para Ascaris lumbricoides e 12,9\% (97/749) para Trichuris trichiura. Desses, dois apresentaram infestação mista por S. mansoni e A. lumbricoides, um por $S$. mansoni e T. trichiura e 65 por $A$. lumbricoides e T. trichiura. No total de amostras de fezes do GC, obteve-se positividade de $16,2 \%$ (6/37) para ovos de A. lumbricoides, 10,8\% (4/37) para larvas de Strongyloides stercoralis e 5,4\% (2/37) para ovos de T. trichiura, sendo que 5,4\% $(2 / 37)$ apresentavam infestação mista por $A$. lumbricoides e T. trichiura.

A positividade sorológica para S. mansoni no GE foi de $36,8 \%$ e $33,5 \%$, respectivamente por ELISA-IgM e RIFI-IgM (Tabela 1). Observou-se concordância quase perfeita, com índice Kappa de 0,89 , quando os resultados obtidos pelos dois métodos sorológicos foram comparados entre

Tabela 1

Positividade sorológica para esquistossomose mansoni, de acordo com o teste sorológico empregado no grupo de estudo. Município de Pedro de Toledo, São Paulo, Brasil, 1998.

\begin{tabular}{|c|c|c|c|}
\hline \multirow[t]{2}{*}{ Testes sorológicos } & \multicolumn{3}{|c|}{ Positividade $(n=591)$} \\
\hline & $\mathrm{n}$ & $\%$ & IC 95\% \\
\hline ELISA-IgM & 218 & 36,8 & $33,0-40,8$ \\
\hline RIFI-IgM & 198 & 33,5 & $29,7-37,5$ \\
\hline
\end{tabular}

si (Tabela 2). Do total de 591 amostras de sangue testadas, 30 revelaram resultados discordantes entre os dois métodos imunológicos utilizados. Para o GC, das amostras de sangue submetidas aos métodos imunológicos, uma foi positiva pelos dois métodos e uma apenas pelo ELISA-IgM.

Dos 12 indivíduos com ovos de S. mansoni nas fezes, 11 foram submetidos à sorologia, oito dos quais foram positivos tanto para ELISA-IgM como para RIFI-IgM. Dos três restantes, duas amostras foram negativas nos dois métodos imunológicos; no exame de fezes verificou-se contagem mínima de oito ovos por grama de fezes (opg), o que equivale à presença de apenas um ovo em uma das três lâminas examinadas. $\mathrm{O}$ terceiro caso apresentou resultado discordante entre ELISA-IgM e RIFI-IgM, sendo positivo apenas nesta última, e o exame de fezes indicou 856 opg.

Os índices de positividade sorológica para esquistossomose mansoni no GE foram analisados de acordo com a presença (positivo) ou não (negativo) de ovos de A. lumbricoides e T. trichiura nas fezes dos 481 indivíduos que forneceram amostras de fezes e de sangue. Observaram-se positividades sorológicas comparáveis nos subgrupos positivos ou negativos para ovos de $A$. lumbricoides, tendo-se verificado o mesmo para ovos de T. trichiura (Tabela 3).

\section{Discussão}

Para este estudo, foi selecionada uma amostra representativa da população do município de Pedro de Toledo. Todas as residências selecionadas foram visitadas. Por causa da dificuldade em encontrar todos os moradores no mesmo momento para coleta de sangue ou para que coletassem as fezes num mesmo dia, não foi possível obter as duas amostras biológicas de todos os indivíduos selecionados. Portanto, foi dado maior enfoque à padronização do método de ELISA-IgM, usando para tanto somente os resultados obtidos dos indivíduos que forneceram as duas amostras biológicas.

Nessa localidade, nos últimos anos, a prevalência da esquistossomose, determinada pelo método parasitológico de fezes, vem se mantendo ao redor de $2 \%$ (Marçal et al., 1999). No presente estudo, o resultado do método parasitológico de fezes indicou positividade de 1,6\% (12/749). A intensidade de infestação, medida pela media geométrica, foi de 40,9opg. Em nove dos 12 portadores de ovos $S$. mansoni nas fezes, a contagem foi inferior a 100opg, com média geométrica de 22,7opg, permitindo classificá- 
los como portadores de infestação leve (WHO, 1997). Em dois casos a contagem foi de 128opg, tendo sido classificados como de infecção moderada. O último portador foi classificado como de infecção intensa, com uma contagem de 856opg.

Os índices de positividade sorológica para esquistossomose foram superiores em mais de 20 vezes aos obtidos pelo método parasitológico de fezes (Tabela 1). Essa falta de concordância observada entre os resultados dos métodos imunológicos e os do parasitológico não foi surpreendente, pois em estudos anteriores realizados em áreas de baixa endemicidade, nos quais se compararam os índices de positividade de métodos imunológicos e parasitológico (Dias et al., 1989; Doenhoff et al., 1993; Eltiro et al., 1992), os primeiros foram de duas a seis vezes superiores aos índices de positividade parasitológica. Cabe considerar que um programa de controle da esquistossomose vem sendo executado na região há mais de 20 anos. De modo geral, nas áreas de aplicação desse programa, pode ser verificado um aumento na discrepância em relação aos índices de positividade parasitológica e sorológica, isto é, à medida que o programa de controle vai sendo aplicado, diminui o índice de positividade parasitológica e aumenta o índice de positividade sorológica. Portanto, incluída nessa positividade sorológica há certamente uma parcela de indivíduos que se curaram com o tratamento ou que se expuseram ao parasita, induziram resposta imune, mas não desenvolveram a doença.

Houve uma concordância quase perfeita entre o ELISA-IgM e a RIFI-IgM (Tabela 2), conforme indicado pelo coeficiente Kappa de 0,89 (IC: 0,84-0,92). Além disso, quando se compararam os níveis de anticorpos IgM detectados pelos dois métodos sorológicos, verificou-se que as amostras que apresentaram maior intensidade de fluorescência pela RIFI-IgM resultaram em maior valor de absorbância pelo ELISA-IgM, sugerindo uma correlação positiva. Isto ocorre, provavelmente, por se tratar de testes sorológicos que revelam anticorpos de mesma classe contra antígenos de natureza polissacarídica presentes ao nível do tubo digestivo do verme, caracterizado por Nash (1978). Estes foram parcialmente purificados (Deelder et al., 1976) e utilizados no ELISA-IgM ou preservados em cortes de vermes adultos e usados na técnica de RIFI-IgM. Entre os 30 casos discordantes (Tabela 2), 25 foram considerados positivos para o método de ELISA-IgM, embora negativos pela RIFI-IgM. Nesses casos, a maioria (19/25) dos valores das médias de absorbância encontrados ficou pouco acima do limiar de reatividade do

\begin{tabular}{|c|c|c|c|}
\hline \multicolumn{4}{|c|}{$\begin{array}{l}\text { Análise comparativa entre os resultados do ELISA-IgM e da RIFI-IgM encontrados } \\
\text { no grupo de estudo. Município de Pedro de Toledo, São Paulo, Brasil, } 1998 .\end{array}$} \\
\hline & \multicolumn{3}{|c|}{ ELISA-IgM } \\
\hline & Positivo & Negativo & Total \\
\hline \multicolumn{4}{|l|}{ RIFI-IgM } \\
\hline Positivo & 193 & 5 & 198 \\
\hline Negativo & 25 & 368 & 393 \\
\hline Total & 218 & 373 & 591 \\
\hline
\end{tabular}

Índice Kappa: 0,89 (0,84-0,92)

Tabela 3

Positividade sorológica para esquistossomose, pelos dois métodos sorológicos, de acordo com a presença (positivo) ou não (negativo) de ovos de Ascaris lumbricoides e Trichuris trichiura, no grupo de estudo. Município de Pedro de Toledo, São Paulo, Brasil, 1998.

\begin{tabular}{|c|c|c|c|c|}
\hline \multirow[t]{3}{*}{ Espécies parasitárias } & \multicolumn{4}{|c|}{ Positividade } \\
\hline & \multicolumn{2}{|c|}{ ELISA-IgM } & \multicolumn{2}{|c|}{ RIFI-IgM } \\
\hline & $\%$ & IC 95\% & $\%$ & IC 95\% \\
\hline \multicolumn{5}{|l|}{ Ascaris lumbricoides } \\
\hline Negativo $(n=371)$ & 37,7 & $32,8-42,9$ & 33,4 & $28,7-38,5$ \\
\hline Positivo $(n=110)$ & 35,5 & $26,7-45,1$ & 30,9 & $22,6-40,5$ \\
\hline \multicolumn{5}{|l|}{ Trichuris trichiura } \\
\hline Negativo $(n=430)$ & 37,7 & $33,1-42,5$ & 33,0 & $28,6-37,7$ \\
\hline Positivo $(n=51)$ & 33,3 & $21,1-48,0$ & 31,4 & $19,5-46,0$ \\
\hline Total $(n=481)$ & 37,2 & $32,9-41,7$ & 32,8 & $28,7-37,3$ \\
\hline
\end{tabular}

dia. Em cinco casos, os resultados da RIFI-IgM foram fracamente positivos, embora as leituras de absorbância no ELISA-IgM tenham ficado abaixo do limiar de reatividade do dia (cutoff). Em termos sorológicos, muitos desses casos poderiam ser considerados negativos; no entanto, é recomendável uma análise individual de cada caso para permitir uma interpretação correta do resultado positivo ou negativo, frente a outros critérios clínicos, epidemiológicos e laboratoriais que cada caso requer.

A determinação da sensibilidade do método em avaliação ficou prejudicada pelo pequeno número de indivíduos positivos no exame de fezes. Dos 12 indivíduos com ovos de S. manso$n i$, apenas em 11 foi possível a coleta de sangue e, destes, três apresentaram-se negativos no ELISA-IgM, resultando em um índice de sensibilidade de $72,7 \%$. Entretanto, ao mesmo tempo 
nossa equipe de pesquisa empregou o ELISAIgM em um grupo de indivíduos comprovadamente esquistossomóticos, mas com baixa carga parasitária, determinada após exaustivas repetições do exame parasitológico de fezes por técnicas de Kato-Katz e eclosão de miracídio em amostras distintas, naqueles pacientes que apresentavam algum sintoma da doença. Nesse grupo o índice de sensibilidade do ELISA-IgM foi de $98 \%$, igual ao encontrado pela técnica de RIFI-IgM (dados submetidos à publicação).

A possibilidade de ocorrência de reações cruzadas entre diferentes espécies de helmintos foi verificada subdividindo-se o GE em quatro subgrupos de acordo com a presença (positivo) ou não (negativos) de ovos de A. lumbricoides e de T. trichiura nas fezes (Tabela 3). Essa análise permitiu observar que a positividade sorológica para esquistossomose não foi maior nos subgrupos positivos para outros helmintos, sugerindo ausência de reações cruzadas, pelo menos entre o $S$. mansoni e essas duas espécies de helmintos, nos métodos imunológicos empregados no presente estudo. Além disso, a bai-

\section{Agradecimentos}

Agradecemos a Maria Cristina Conceição de Mello pelo apoio técnico na manutenção do ciclo do S. manso$n i$ è equipe da Superintendência de Controle de Endemias pela coleta de material no campo. Ao Conselho Nacional de Desenvolvimento Científico e Tecnológico pela bolsa de estudos e à Fundação de Amparo à Pesquisa do Estado de São Paulo pelo auxílio financeiro parcial (Processo 97/13904-0).

\section{Referências}

BARRETO, M. L.; SMITH, D. H. \& SLEIGH, A. C., 1990. Implications of faecal egg count variation when using the Kato-Katz method to assess Schistosoma mansoni infections. Transactions of the Royal Society of Tropical Medicine and Hygiene, 84:554555.

CERQUEIRA, F. L., 1988. Coprotest: Metodologia confiável para o exame parasitológico de fezes. $L A E S$ Laboratórios: Administração, Produtos, Serviços e Equipamentos para Laboratórios de Análises Clínicas, Patológicas e Radiologia, 9:5-12.

CORREIA-OLIVEIRA, R.; DUSSE, L. M.; VIANA, I. R.; COLLEY, D. G.; SANTOS CARVALHO, O. \& GAZZINELLI, G., 1988. Human antibody response against schistosomal antigens. I. Antibodies from patients with Ancylostoma, Ascaris lumbricoides or Schistosoma mansoni infections react with schisto- xa positividade sorológica encontrada no GC sugere boa especificidade do método de ELISAIgM. Nesse grupo, embora composto de indivíduos fortemente parasitados por espécies de helmintos, como A. lumbricoides, T. trichiura e S. stercoralis, apenas dois indivíduos apresentaram-se positivos para ELISA-IgM e um para RIFI-IgM, conferindo boa especificidade para o método sorodiagnóstico em questão.

Finalmente, a concordância quase perfeita encontrada quando se analisaram comparativamente os resultados do ELISA-IgM e da RIFIIgM - ressaltando-se que esta última vem apresentando bons índices de sensibilidade e especificidade (Kanamura et al., 1998a, 1998b; Lima et al., 1998) - e a ausência de reações cruzadas quanto aos parasitas encontrados pelo método parasitológico de fezes nos indivíduos do GE e do GC, sugerem que o ELISA-IgM, padronizado e avaliado em nossas condições laboratoriais e ambientais, constitui-se em um método potencialmente útil para fins diagnósticos da esquistossomose, em indivíduos com baixa carga parasitária. some antigens. American Journal of Tropical Medicine and Hygiene, 38:348-355.

DEELDER, A. M.; KLAPPE, H. T. M.; VAN DER AARDWEG, G. J. M. J. \& VAN MEERBERG, E. H. E. M., 1976. Schistosoma mansoni: Demonstration of two circulating antigens in infected hamsters. Experimental Parasitology, 40:189-197.

DEELDER, A. M. \& KORNELIS, D., 1980. A comparation of IFA an ELISA for the demonstration of antibody against schistosome gut-associated polysaccharide antigen in schistosomiasis. Zeitschrift fur Parasitenkunde, 64:65-75.

DE VLAS, S. J. \& GRYSSELS, B., 1992. Underestimation of Schistosoma mansoni prevalences. Parasitology Today, 8:274-277.

DIAS, L. C. S.; KAWAZOE, U.; GLASSER, C. M.; HOSHINO-SHIMIZU, S.; KANAMURA, H. Y.; CORDEIRO, 
J. A.; GUARATA, O. F. \& ISHIHATA, G. J., 1989. Schistosomiasis mansoni in the municipality of Pedro de Toledo (São Paulo, Brasil) where the Biomphalaria tenagophila is the snail host: I. Prevalence in human population. Revista do Instituto de Medicina Tropical de São Paulo, 31:110-118.

DIAS, L. C. S.; MARÇAL Jr., O.; GLASSER, C. M.; KANAMURA, H. Y. \& HOTTA, L. K., 1992. Control of schistosomiasis mansoni in a low transmission área. Memórias do Instituto Oswaldo Cruz, 87:233239.

DOENHOFF, M. J.; BUTTERWORTH, A. E.; HAYES, R. J.; STURROCK, R. F; OUMA, J. H.; KOECH, D.; PRENTICE, M. \& BAIN, J., 1993. Seroepidemiology and serodiagnosis in Kenya using crude and purified egg antigens of Schistosoma mansoni in ELISA. Transactions of the Royal Society of Tropical Medicine and Hygiene, 87:42-48.

ELTIRO, F; YE-EBIYO, Y. \& TAYLOR, M. G., 1992. Evaluation of an enzyme linked immunosorbent assay (ELISA) using Shistosoma mansoni soluble egg antigen as a diagnostic tool for Shistosoma mansoni Ethiopian choolchildren. Journal of Tropical Medicine and Hygiene, 95:52-56.

ENGELS, D.; SINZINKAYO, E. \& GRYSEELS, B., 1996. Day-to-day egg count fluctuation in Schistosoma mansoni infection and its operational inplications. American Journal of Tropical Medicine and Hygiene, 54:319-324.

FERREIRA, C. S. \& CARVALHO, M. E., 1982. Padronização de uso de papel-filtro como suporte de material para reações sorológicas. Revista Brasileira de Malariologia, 34:82-86.

KAMAL, K. A.; SHAHEEN, H. I. \& EL-SAID, A. A., 1994. Applicability of Elisa on bufferr-eluates of capillary blood spotted on filter papers for the diagnosis and clinical staging of human schistosomiasis. Tropical and Geographical Medicine, 46:138-141.

KANAMURA, H. Y.; DIAS, L. C. S.; SILVA, R. M.; GLASSER, C. M.; PATUCCI, R. M. J.; BELLOSA, S. A. G. \& ANTUNES, J. L. F., 1998a. A comparative epidemiologic study of specific antibodies (IgM and IgA) and parasitological findings in an endemic area of low transmission of Schistosoma mansoni. Revista do Instituto de Medicina Tropical de São Paulo, 40:85-91.

KANAMURA, H. Y.; DIAS, L. C. S.; GLASSER, C. M.; SILVA, R. M.; PATUCCI, R. M. J.; CHIODELLI, S. G. \& ADDISS, D. G., 1998b. Detection of IgM antibodies to Schistosoma mansoni gut-associated antigens for the study of the dynamics of schistosomiasis transmission in na endemic area with low worm burden. Revista do Instituto de Medicina Tropical de São Paulo, 40:225-231.

KATZ, N.; CHAVES, A. \& PELLEGRINO, J., 1972. A simple device for quantitative stool thrick smear technique in schistosomiasis mansoni. Revista do Instituto de Medicina Tropical de São Paulo, 14:397400.

LIMA, V. L. C.; GUERCIO, V. M. F.; RANGEL, O.; KANAMURA, H. Y. \& DIAS, L. C. S., 1998. Immunofluorescence test on Schistosoma mansoni worm paraffin sections (IgM-IFT) for the study of schistosomiasis transmission in Campinas, São Paulo, Brazil. Memórias do Instituto Oswaldo Cruz, 93: 283-288.
MARCCAL Jr., O.; HOTTA, L. K.; PATUCCI, R. M. J.; GLASSER, C. M. \& DIAS, L. C. S., 1993. Schistosomiasis mansoni in an area of low transmission. II Risk factors for infection. Revista do Instituto de Medicina Tropical de São Paulo, 35:331-335.

MARÇAL Jr., O.; DIAS, L. C. S.; KANAMURA, H. Y.; SOARES, L. C. B. \& OLIVEIRA, E. J., 1999. Evaluation of a shistosomiasis mansoni control programme in an area of low endemicity, in Brazil. In: VII International Symposium on Shistosomiasis, Abstracts, p. 79, Rio de Janeiro: Fundação Oswaldo Cruz.

NASH, T. E., 1978. Antibody response to a polysaccharide antigen present in the schistosome gut. American Journal of Tropical Medicine and Hygiene, 27:938-943.

NOYA, B. A.; BALZAN, C.; ARTEAGA, C.; CESARI, I. \& NOYA, O., 1999. The last fifteen years of schistosomiasis in Venezuela: Features and evolution. Memórias do Instituto Oswaldo Cruz, 94:139-146.

SATHE, B. D.; PANDIT, C. H.; CHANDERKAR, N. G.; BADADE, D. C.; SENGUPTA, S. R. \& RENAPURKAR, D. M., 1991. Serodiagnosis of schistosomiasis by ELISA test in an endemic area of Gimvi Vilage, India. Journal of Tropical Medicine and Hygiene, 94: 76-78.

SILVA, R. M.; SILVA, M. I. P. G.; VELLOSA, S. A. G. \& KANAMURA, H. Y., 1992. Pesquisa de anticorpos IgM contra tubo digestivo do verme para diagnóstico da esquistossomose mansônica. Revista Brasileira de Patologia Clínica, 28:39-42.

SILVA, R. M.; KANAMURA, H. Y.; CAMARGO, E. D.; CHIODELLI, S. G.; NAKAMURA, P. M.; GARGIONI, C.; VELLOSA, S. A. G. \& ANTUNES, J. L. F., 1998. A comparative study on IgG-ELISA, IgM-IFT and Kato-Katz methods for epidemiological purposes in a low endemic area for schistosomiasis. Memórias do Instituto Oswaldo Cruz, 93:279-282.

TANABE, M.; OKAZAKI, M.; KOBAYASHI, S.; KANEKO, N.; SEKIGUCHI, T.; TATENO, S.; MOTA, S. R. N. \& TAKEUCHI, T., 1990. Serological studies on schistosomiasis in the Northeast Brazil. Revista do Instituto de Medicina Tropical de São Paulo, 32:121131.

VALLI, L. C. P.; KANAMURA, H. Y.; SILVA, R. M.; SILVA, M. I. P. G.; VELLOSA, S. A. G. \& GARCIA, E. T., 1997. Efficacy of an enzyme-linked immunosorbent assay in the diagnosis of and serologic distinction between acute and chronic Schistosoma mansoni infection. American Journal of Tropical Medicine and Hygiene, 53:358-362.

WHO (World Health Organization), 1997. Schistosomiasis In-Progress 1995-1996: Thirteenth Programme Report of the UNDP/World Bank/WHO Special Programme for Research and Training Diseases. Geneva: WHO.

Recebido em 17 de janeiro de 2002

Versão final reapresentada em 27 de maio de 2002

Aprovado em 28 de agosto de 2002 